Artigo

\title{
Predicción Climática Estacional de Precipitación Acumulada en Primavera y Verano en el Sur de Uruguay
}

\author{
Matilde Ungerovich, Marcelo Barreiro \\ Departamento de Ciencias de la Atmósfera de Universidad de la República, \\ Montevideo, Uruguay.
}

Recebido em 29 de Abril de 2015 - Aceito em 15 de Dezembro de 2015

\begin{abstract}
Resumen
Se desarrollan diferentes modelos pre-operativos de pronóstico dinámico-estadístico de precipitación estacional en el sur de Uruguay para primavera y verano. Para ello se utilizan regresiones lineales entre predicciones de variables dinámicas y observaciones de precipitación. Los pronósticos se inicializan en Agosto y Noviembre para los trimestres de Setiembre-Noviembre y Diciembre-Febrero, respectivamente. Las predicciones de las variables dinámicas son salidas de un ensamble del modelo de circulación general de la atmósfera ICTP MCGA forzado con condiciones de borde de temperatura de superficie del mar (TSM) pronosticadas por NCEP-CFSv2. Las observaciones de precipitación provienen de 10 estaciones meteorológicas ubicadas al sur del Río Negro. Los mejores índices predictores se encuentran mediante validación cruzada, utilizando ventanas de un año, con las variables dinámicas. Se concluye que el mejor índice predictor es el viento meridional en $200 \mathrm{hPa}$ promediado en una región que incluye el Sudeste de Sudamérica y es tal que anomalías de componente norte están asociadas a lluvias por encima de lo normal en el sur del país. Se encuentra que para todo el sur del país los pronósticos tienen habilidad únicamente en primavera mientras que para la zona metropolitana de Montevideo los pronósticos muestran habilidad para ambas estaciones y principalmente para verano.
\end{abstract}

Palabras-clave: Predicción estacional, precipitación.

\section{Seasonal Forecast of Accumulated Rainfall in Southern Uruguay During Spring and Summer}

\begin{abstract}
We develop dynamical-statistical forecast models in order to predict seasonal rainfall in southern Uruguay during summer and spring. The statistical technique consists of linear regressions between dynamic variables and rainfall observations. The forecasts for September-October-November are initialized in August and the ones for December-January-February in November. The dynamic variables are ICTP-MGCAs outputs, forced with sea surface temperature, predicted by NCEP-CFSv2, as boundary conditions. The observational data is accumulated monthly, values are measured in 10 meteorological stations that are cross-correlated, using one year windows, with the dynamic variables in order to find the best predictor indexes. We conclude that the best predictor index is the meridional wind in the $200 \mathrm{hPa}$ level averaged in an area that includes the Southeast of South America. Northern wind anomalies in this area are associated with positive rainfall anomalies in Southern Uruguay. We found that while forecasts for the south of the country are skillful only in spring, forecasts for the metropolitan area of Montevideo are skilfull in both seasons showing their best performance in summer.
\end{abstract}

Keywords: southern Uruguay, seasonal forecast, rainfall.

\section{Introducción}

La economía de Uruguay se basa en el aprovechamiento de sus recursos naturales, ya sea mediante agricul- tura, ganadería o turismo. Debido a la naturaleza económica del país se torna imprescindible conocer un pronóstico climático estacional preciso. En particular, las condiciones atmosféricas de las estaciones de primavera y verano jue-

Autor de correspondência: Matilde Ungerovich,mungerovich@fisica.edu.uy. 
gan un papel importante, especialmente la precipitación acumulada.

La predicción del clima puede realizarse únicamente en determinadas regiones y épocas del año con algunos meses de antelación. La zona definida por el sur de Brasil, noreste de Argentina y Uruguay tiene gran correlación con la temperatura de superficie del mar (TSM) en el Océano Pacífico Ecuatorial (por ejemplo Ropelewski y Halpert, 1987, Grimm et al., 2000). La variabilidad climática en esta zona está determinada por la influencia de El Niño Oscilación Sur (ENOS) y por la variabilidad intrínseca de la dinámica atmosférica. Si bien la variabilidad intrínseca no puede predecirse con gran antelación, la influencia de ENOS aumenta el grado de predictibilidad climática (Barreiro, 2010).

Varios estudios prueban correlaciones significativas entre anomalías de precipitación acumulada en el sudeste de Sudamérica y las fases extremas de ENOS. Barreiro (2010) demuestra que existe gran predictibilidad en la zona denominada SESA (Southeastern South America) comprendida entre las longitudes $65^{\circ} \mathrm{O}-47^{\circ} \mathrm{O}$ y las latitudes $19^{\circ} \mathrm{S}-37^{\circ} \mathrm{S}$ durante las estaciones y períodos en las cuales ENOS tiene influencia. Algunos estudios (por ejemplo Cazes-Boezio et al., 2012) analizan métodos para mejorar la calidad de los pronósticos climáticos estacionales para el norte de Uruguay, región del país con mayor influencia de ENOS. Sin embargo, pocos (por ej. Pisciottano et al., 1994) estudian la relación entre ENOS y la precipitación en el sur.

El objetivo general de este trabajo es desarrollar un modelo dinámico-estadístico que mejore la calidad del pronóstico numérico de la media de precipitación acumulada estacional, calculado con un mes de antelación a la estación de interés, en el sur de Uruguay (considerado como la región ubicada al sur del Río Negro que se encuentra aproximadamente en $32.5^{\circ} \mathrm{S}$ ) para primavera y verano. Para la parte dinámica del modelo se utilizará el modelo ICTP MCGA, mientras que en la parte estadística se buscarán relaciones entre la precipitación y variables dinámicas.

Consideraremos como factores influyentes en la precipitación los vientos en 200 y $850 \mathrm{hPa}$. Variaciones en la circulación de capas altas afectan el desarrollo de centros de baja presión en superficie a través de la advección de vorticidad ciclónica. Al mismo tiempo, dado que las anomalías de circulación forzadas por ENOS en la región extratropical son fundamentalmente barotrópicas (Horel y Wallace, 1981), las anomalías observadas en $200 \mathrm{hPa}$ son representativas de los cambios en toda la columna. Por otro lado, los vientos en capas bajas son los responsables de la advección de humedad desde el norte, fundamental para mantener el régimen de precipitación en la región.

El artículo está estructurado de la siguiente forma: en la segunda sección se detallan los datos y las técnicas utilizadas, en la sección 3 se explica la metodología, en la sección 4 se muestran y discuten resultados y la última sección se centra en las conclusiones.

\section{Datos y Técnica Utilizada}

\subsection{Datos}

Se utilizan datos observacionales, de reanálisis y pronósticos retrospectivos.

El análisis de los datos se hace en forma de anomalías sin tendencia (con respecto al período 1991-2011). A la hora de separar los datos estacionalmente, se trabaja con anomalías de medias trimestrales. Se considera al verano como Diciembre, Enero y Febrero y se lo cataloga como el verano del año de los meses de Enero y Febrero. Es decir, el verano del año 2000 es la media de las anomalías de Diciembre de 1999, Enero de 2000 y Febrero de 2000. Por otro lado, primavera de cierto año es la media de las anomalías de Setiembre Octubre y Noviembre del mismo.

La base de datos observacionales consiste en acumulados mensuales de precipitación observada en las estaciones del Instituto Uruguayo de Meteorología (DNMINUMET). Se cuenta con datos en el período 1991-2011 de 10 estaciones meteorológicas ubicadas en la zona sur (Fig.1). La interpolación de los mapas de observación se realiza mediante la técnica krigging (Stein, 2012).

Se utilizan los datos de reanálisis del proyecto ERAInterim (Uppala et al., 2008) de las variables TSM y viento en los niveles de $200 \mathrm{hPa}$ y $850 \mathrm{hPa}$. La resolución horizontal es de $1.5^{\circ}$. Los datos se descargaron de ECMWF Data Server (Dee et al., 2011). Estos datos de reanálisis se usaron para validar la variabilidad de la circulación atmosférica simulada por el modelo dinámico utilizado.

Por último, se cuenta con las salidas del modelo de pronóstico NCEP-CFSv2 (Saha et al., 2014) para TSM que se descargan de la librería de datos de IRI. CFSv2 es un modelo climático acoplado, implementado por el National Center for Environmental Prediction (NCEP). La resolución espacial atmosférica es T126 (100 km x $100 \mathrm{~km})$. El modelo oceánico es MOM4 con resolución espacial meridional $0.25^{\circ}$. En particular, en este trabajo, se utilizan los primeros 12 miembros del ensamble con una antelación máxima de los pronósticos retrospectivos de 3 meses. Los pronósticos retrospectivos que se utilizan son los calculados en el período 1991-2010. Entonces, se cuenta con pronósticos de TSM para los meses Setiembre, Octubre, Noviembre y Diciembre desde 1991 hasta 2010 y para Enero y Febrero desde 1992 hasta 2011.

\subsection{Técnica utilizada}

La técnica utilizada para mejorar la calidad del pronóstico estacional de la precipitación (PP) media acumulada es una anidación entre un Modelo de Circulación General de la Atmósfera (MCGA) y diferentes modelos estadísticos, entre los cuales se elegirán los de mayor habilidad. El modelo dinámico es el ICTP-MCGA ó Speedy ("Simplified Parameterizations, privitivE-Equation Dynamics") (Molteni, 2003, Kucharski et al., 2006, Kucharski et al., 2013). Cuenta con 8 niveles verticales “completos" y 


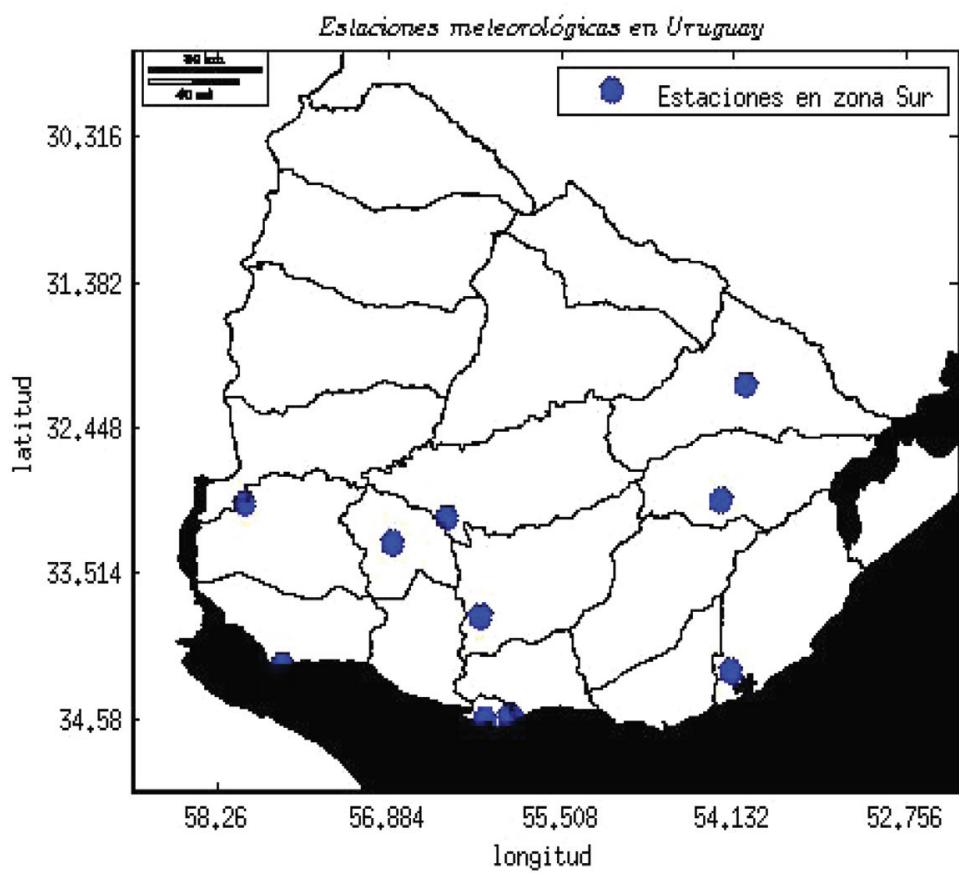

Figura 1 - Distribución espacial de las estaciones meteorológicas de DNM-INUMET de la zona sur.

8 niveles "medios". La resolución horizontal del modelo es $3.75^{\circ} \times 3.75^{\circ}$.

Los modelos estadísticos utilizados son diferentes regresiones lineales simples y múltiples entre la variable a predecir e índices de vientos en 200 y $850 \mathrm{hPa}$ construidos a partir de las salidas del MCGA. Para obtener los parámetros de las regresiones es necesario contar con un período de calibración, en el cual tanto predictor como predictando son conocidos. En este trabajo se procedió mediante validación cruzada con el período de calibración Setiembre 1991Febrero 2011, dejando afuera al año que se predice.

\section{Metodología}

La metodología de trabajo se divide en tres etapas. En la primer etapa se realiza un estudio de predictibilidad, que apunta a encontrar las zonas en las cuales el modelo dinámico es capaz de representar adecuadamente la evolución observada de algunas variables. La segunda etapa es la construcción de los modelos estadísticos. Finalmente, la tercer etapa es la validación de los modelos anidados.

\subsection{Primera etapa: estudio de la habilidad del modelo dinámico}

El estudio de la predictibilidad del modelo dinámico se basa en la comparación de los datos de reanálisis con las salidas del MCGA. Se trabaja con ensambles de 10 miembros generados a partir de diferentes condiciones iniciales de la atmósfera. Los pronósticos se inicializan en Agosto y Noviembre y tienen una antelación máxima de 3 meses.

Conocimientos previos y análisis de las salidas muestran que el modelo tiene su mayor habilidad en la descrip- ción del campo de vientos. Por lo tanto, para analizar la habilidad del modelo se comparan los mapas de correlación entre las anomalías de precipitación media estacional observada promediada entre las estaciones meteorológicas del sur de Uruguay y reanálisis de viento y vientos pronosticados por el MCGA. Se considera que el modelo tiene habilidad (existiendo predictibilidad) en las regiones y estaciones del año en las cuales estos dos mapas son similares.

\subsection{Segunda etapa: generación del modelo estadístico}

La etapa de generación del modelo estadístico comienza por encontrar, entre las variables que el modelo pronostica hábilmente en las zonas y estaciones del año en las que esto sucede, correlaciones significativas con las anomalías de precipitación promediada en el sur de Uruguay y en la estación meteorológica de Carrasco, ubicado en la zona metropolitana de Montevideo (que se estudia como caso particular). Además, estas correlaciones significativas (que superan el nivel de confianza de $90 \%$ en un test de student de dos colas) deben tener un significado físico que las respalde. Los promedios espaciales de las variables seleccionadas (vientos en diferentes niveles de presión), pronosticadas por el MCGA, en dicha zona serán los predictores. Los parámetros de las regresiones lineales simples se obtienen cruzando las series temporales de variable independiente con las del predictando en el período de calibración. Luego, para calcular los parámetros de las regresiones lineales múltiples se repite el proceso con todas las combinaciones posibles de predictores. Esto se realiza para primavera y verano.

Una vez que se tienen los parámetros de las regresiones se procede a la generación específica de los pronósti- 
cos. Se implementan pronósticos probabilísticos y determinísticos. El pronóstico determinístico consiste en calcular, con los parámetros de regresiones lineales simples y múltiples hallados, el valor de la variable dependiente del año a predecir (precipitación), en función de las medias de los ensambles de los predictores. Por otro lado, para el pronóstico probabilístico se repite el proceso para cada miembro del ensamble de predictores y se calculan porcentajes. En ambos casos, se expresan los resultados en midiles y terciles segun percentiles climatológicos del período de estudio.

\subsection{Tercer etapa: validación de los modelos}

Se utilizan dos métodos de validación para los modelos determinísticos y uno para los modelos probabilísticos. Para cada método de validación se define un nivel de aceptabilidad, a partir del cual el resultado se considera bueno.

El primer método de evaluación de modelos determinísticos es la correlación lineal entre la anomalía de precipitación pronosticada retrospectivamente para cada año del período de calibración y la observada. Se analiza la significancia estadística de las correlaciones de Pearson y Spearman comparando los valores con un test de student de 2 colas con un nivel de significancia de $90 \%$.

En segundo lugar, se utiliza una validación de porcentaje de aciertos. Esto es una adaptación del Heidke Score (Mason, 2013) a un modelo de pronóstico determinístico. El método consiste en calcular el porcentaje de veces en las cuales el pronóstico y la observación coincidieron en los cuantiles climáticos. Para determinar la validez del modelo se considera que esta medida sigue una distribución binomial (con probabilidad de 0.50 en midiles y 0.33 en terciles) y se compara con el nivel de significancia de $90 \%$. Entonces, para el caso de estudio, donde se considera al pronóstico para cada año como un ensayo independiente, el porcentaje de aciertos en midiles es aceptable cuando supera el $75 \%$ y en terciles cuando supera el 55\%.

El método de validación para pronósticos probabilísticos es el Brier Score Original (BS) (Wilks, 2011), definido como: $B S=\frac{1}{N} \sum_{t=1}^{N} \sum_{i=1}^{R}\left(f_{t i}-o_{t i}\right)^{2}$ donde R es la cantidad de casos posibles (primer, segundo o tercer tercil), $\mathrm{N}$ es la cantidad de predicciones (uno para cada miembro del ensamble), $\mathrm{f}_{\mathrm{t}}$ es la probabilidad adjudicada a cada una de las posibles situaciones y $\mathrm{o}_{\mathrm{t}}$ es 1 para la situación ocurrida y 0 en las demás. Para definir si el valor es aceptable se compara con los valores climatológicos, entonces, los BS buenos en midiles son los inferiores a 0.500 mientras que en terciles son los inferiores a 0.667 .

\section{Resultados y Discusión}

\subsection{Selección de índices}

De acuerdo a la primera y segunda etapa de la metodología se eligieron tres predictores para cada estación.
Para ello se delimitaron las regiones variando las posiciones y tamaños de las zonas para que su promedio espacial mantenga la máxima correlación posible con la precipitación tanto en el sur de Uruguay como en la estación de la zona metropolitana de Carrasco. En particular, se eligieron dos predictores en el nivel de $200 \mathrm{hPa}$ y uno en el de 850 hPa (Fig. 2).

La relación física entre los predictores seleccionados y las lluvias ha sido estudiada por varios autores. En primer lugar, se destaca que las correlaciones observadas en el nivel de $200 \mathrm{hPa}$ con circulación ciclónica al oeste y anticiclónica al este de América del Sur (que en verano aparecen como una cuña) se corresponden con los principales modos de variabilidad interanual que encuentran Robertson y Mechoso (2000). Las anomalías de circulación al este de Sudamérica también coinciden con los resultados de Zamboni et al. (2010). En particular, Zamboni et al. (2010), encuentran este vórtice como modo principal de variabilidad interanual de los vientos promediados en Octubre y Noviembre y como segundo modo de los vientos promediados en Enero y Febrero. Encuentran también que el vórtice es casi barotrópico en primavera, verano y otoño y que en estas estaciones mantiene una relación lineal simultánea con las anomalías de precipitación en SESA. Por otro lado, Robertson y Mechoso (2000) encuentran como primer modo en un análisis de Funciones Empíricas Ortogonales (EOF 1) un ciclón centrado en $45^{\circ} \mathrm{O}-30^{\circ} \mathrm{S}$ con una extensión latitudinal entre $50^{\circ} \mathrm{S}$ y $10^{\circ} \mathrm{S}$ y longitudinal entre $70^{\circ} \mathrm{O}$ y $20^{\circ} \mathrm{O}$ que explica el $23 \%$ de la varianza total durante DiciembreEnero-Febrero. El EOF 2 también presenta una circulación ciclónica centrada en $40^{\circ} \mathrm{O}-25^{\circ} \mathrm{S}$ pero está dominado por vientos del oeste al norte de $15^{\circ} \mathrm{S}$ y por un ciclón centrado al este de la Patagonia ( $16 \%$ de la varianza). Por último, Cazes-Boezio et al. (2003) también encuentran que el EOF 1 promediado en Octubre-Noviembre-Diciembre consiste en un vórtice anticiclónico centrado en SESA y vientos oeste al norte de América del Sur.

Además, varios autores han conectado la existencia del anticiclón al este de Uruguay con ENOS. Por ejemplo, Zamboni et al. (2010) encuentran correlaciones significativas entre el EOF 1 de Octubre y Noviembre y el EOF 2 de Enero y Febrero con vientos en el Pacífico Sur en $200 \mathrm{hPa}$ que están, a su vez, asociados con el principal patrón de comportamiento del Océano Pacífico Sur, conocido como Pacific-South America Pattern (PSA 1), el cual se asocia con las anomalías de TSM en el Pacífico Central y Este. Asimismo, Cazes-Boezio et al. (2003) encuentran que el EOF 1 de vientos en $200 \mathrm{hPa}$ sobre Sudamérica (que incluye el anticiclón) en primavera está correlacionado positivamente con el Pacífico Ecuatorial. Por otro lado, en Enero-Febrero, encuentran que el anticiclón aparece en el EOF 2 y está muy débilmente correlacionado con el Pacífico Ecuatorial. Por otro lado, Robertson y Mechoso (2000) relacionan el anticiclón encontrado en el EOF 1 con una 

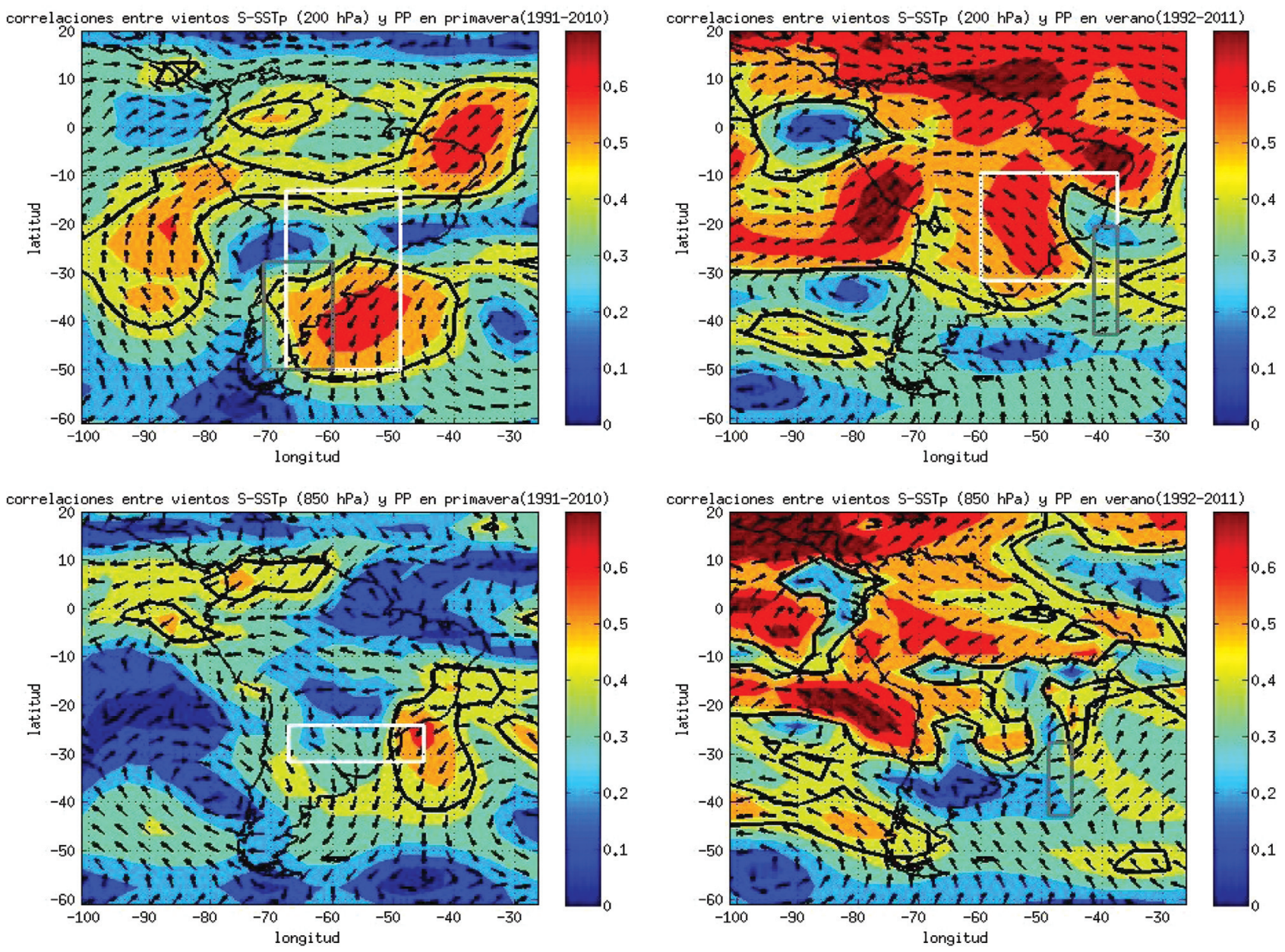

Figura 2 - Correlaciones entre PP y salidas de vientos con TSM pronosticados y zonas seleccionadas como predictores, en el nivel de $200 \mathrm{hPa}$ (imágenes superiores) y en el nivel de 850 (imágenes inferiores), en primavera (izquierda) y en verano (derecha). Las regiones delimitadas con contorno negro son las que se correlacionan significativamente con PP en el sur de Uruguay. Las regiones delimitadas en blanco corresponden a los índices meridionales y las delimitadas en gris a las de los índices zonales.

onda de Rossby estacionaria, y el patrón de vientos del EOF 2 con ENOS.

El hecho de que los patrones de vientos en altura correlacionados con las precipitaciones al sur de Uruguay sean similares a los modos de variabilidad que se relacionan con ENOS implica predictibilidad potencial de las lluvias.

Al analizar la relación física entre los vientos y las precipitaciones, Zamboni et al. (2010) proponen que, como el vórtice anticiclónico es casi barotrópico, las anomalías de viento en capas altas implican un aumento en la advección de humedad en capas bajas desde el norte. Por otro lado, Grimm et al. (2000) encuentran anomalías de circulación casi barotrópicas sobre los Océanos Pacífico y Atlántico durante eventos ENOS. Durante El Niño (La Niña) la anomalía de circulación es ciclónica (anticiclónica) al oeste de SESA y anticiclónica (ciclónica) al este. Los autores relacionan estos dipolos con anomalías de precipitación sobre SESA debido a la combinación de advección de vorticidad ciclónica en capas altas y la advección meridional de humedad en niveles bajos. En las imágenes de verano en el nivel de $200 \mathrm{hPa}$ (Fig. 2) se observa la correlación con una circulación en forma de vaguada (cuña) al oeste (este) de Uruguay, lo cual implica flujo supergeostrófico (subgeostrófico). Esto significa que el viento ageostrófico tiene el mismo sentido (sentido opuesto) que el viento geostrófico, implicando advección de vorticidad ciclónica y divergencia en altura sobre Uruguay (por ejemplo Holton, 2013). La divergencia en altura crea condiciones propicias para la convergencia en superficie, bajas presiones y precipitación.

De acuerdo a la discusión anterior, para definir un índice de velocidad meridional en altura, se elige una región con correlaciones medias espaciales significativas en la componente norte que incluya SESA tanto para primavera como para verano. Por otro lado, la zona seleccionada para el viento zonal (U) en $200 \mathrm{hPa}$ para primavera está relacionada fundamentalmente con el ciclón al oeste de Uruguay, mientras que en verano está asociada al cambio en la intensidad del jet subtropical. 
En el nivel de $850 \mathrm{hPa}$ se considera un índice que represente el fenómeno de advección de humedad meridional. En primavera se considera el promedio espacial del viento meridional al norte de Uruguay. En verano se promedian los vientos zonales en la región seleccionada, la cual forma parte del anticiclón situado sobre SESA y pretende representar tanto la advección de humedad del noroeste como una zona frontal entre aire cálido y frío que también puede ser causa de precipitación.

\subsection{Relación de los índices elegidos con las lluvias al sur de Uruguay}

La Tabla 1 muestra los valores y niveles de significancia estadística de las correlaciones entre los índices seleccionados y las anomalías de precipitación al sur de Uruguay. Se considera no significativo a cualquier valor inferior al límite de significancia previamente mencionado.
Se encuentran tres índices estadísticamente significativos en primavera y dos en verano. A pesar de que el índice U 850 no mantiene correlaciones significativas con la precipitación en el sur, se decide proceder con el estudio ya que, genera buenos resultados cuando se considera únicamente la estación ubicada en la zona metropolitana de Montevideo (Carrasco). Por otro lado, se destaca que V 200 mantiene las mayores correlaciones en las dos estaciones.

\subsection{Validación de los modelos anidados}

\subsubsection{Validación por correlación de los modelos} determinísticos

La Fig. 3 muestra los gráficos de algunos de los pronósticos retrospectivos obtenidos con los modelos construidos usando diferentes índices junto con las observaciones para cada año en primavera.

También se muestran, en la Tabla 2, los valores de las correlaciones entre las anomalías de precipitación pronos-

Tabla 1 - Correlaciones de Pearson y Spearman entre índices y precipitación en el sur y sus niveles de significancia.

\begin{tabular}{lcccc}
\hline Variable independiente & Correlación de Pearson & Significancia & Correlación de Spearman & Significancia \\
\hline U 200 hPa primavera & -0.394 & $91 \%$ & -0.400 & $92 \%$ \\
V 200 hPa primavera & -0.597 & $99 \%$ & -0.528 & $99 \%$ \\
V 850 hPa primavera & -0.493 & $98 \%$ & -0.393 & $91 \%$ \\
U 200 hPa verano & 0.471 & $96 \%$ & 0.403 & $92 \%$ \\
V 200 hPa verano & -0.488 & $98 \%$ & -0.474 & $96 \%$ \\
U 850 hPa verano & 0.286 & NO & 0.256 & NO \\
\hline
\end{tabular}

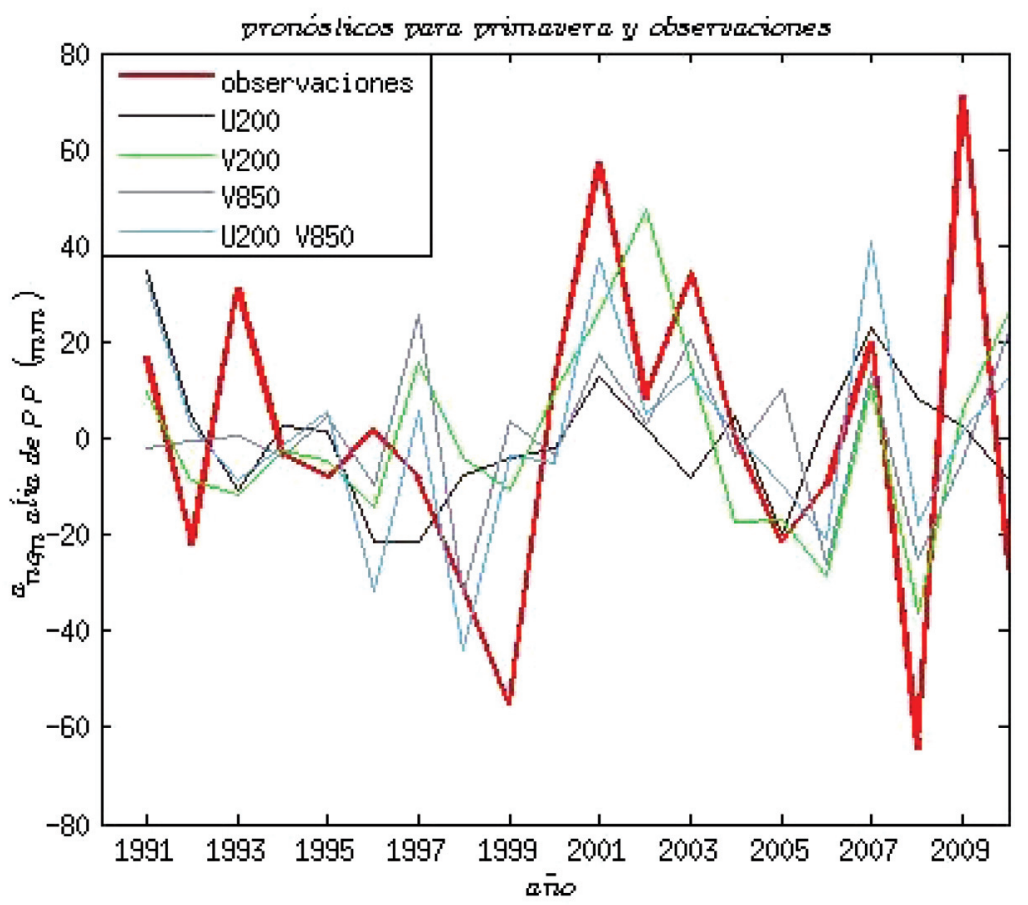

Figura 3 - Pronósticos y observaciones para primavera. 
Tabla 2 - Correlaciones y niveles de significancia estadística entre pronósticos y observaciones en el sur.

\begin{tabular}{lcccc}
\hline Variable independiente & Correlación de Pearson & Significancia & Correlación de Spearman & Significancia \\
\hline U 200 primavera & 0.216 & NO & 0.180 & NO \\
V 200 primavera & 0.465 & $96 \%$ & 0.417 & $94 \%$ \\
V 850 primavera & 0.321 & NO & 0.182 & NO \\
U 200 V 850 primavera & 0.474 & $96 \%$ & 0.466 & $97 \%$ \\
U 200 verano & 0.236 & NO & 0.218 & NO \\
V 200 verano & 0.291 & NO & 0.424 & $94 \%$ \\
U 850 verano & -0.070 & NO & 0.048 & NO \\
V 200 U 850 verano & 0.221 & NO & 0.298 & NO \\
\hline
\end{tabular}

ticada y observada para primavera y verano para los modelos que ofrecen los mejores pronósticos.

La única variable individual cuyo pronóstico se correlaciona significativamente con la observación es V 200 en primavera. A pesar de que en verano V 200 no es estadísticamente significativo según la correlación de Pearson, es el índice que mantiene las mayores correlaciones. Además, V 200 es el índice que tiene correlaciones más altas con las precipitaciones en ambas estaciones (ver Tabla 1).

Se destaca que en primavera, a pesar de que los pronósticos utilizando V 850 y U 200 como predictores individuales no mantienen correlaciones significativas con las observaciones, el modelo que predice con la combinación de ambos índices ofrece los mejores resultados. Esto implica que V 850 y U 200 aportan información complementaria. Por el contrario, en verano el modelo que predice con combinación de predictores no supera a la mayor correlación de los modelos de predictores individuales.

\subsubsection{Validación por porcentaje de acierto de los modelos determinísticos}

A continuación se muestran, en la Tabla 3, los resultados obtenidos en la validación por porcentaje de acierto. Se recuerda que para el caso de estudio (20 ensayos inde-

Tabla 3 - Porcentaje de aciertos de los modelos determinísticos. En negrita los valores buenos.

\begin{tabular}{lcc}
\hline Predictor & $\begin{array}{c}\text { Porcentaje de } \\
\text { aciertos- midiles }\end{array}$ & $\begin{array}{c}\text { Porcentaje de } \\
\text { aciertos- terciles }\end{array}$ \\
\hline U 200 primavera & $55 \%$ & $40 \%$ \\
V 200 primavera & $\mathbf{7 5 \%}$ & $45 \%$ \\
V 850 primavera & $45 \%$ & $50 \%$ \\
U 200 V 850 primavera & $60 \%$ & $\mathbf{6 5 \%}$ \\
U 200 verano & $65 \%$ & $40 \%$ \\
V 200 verano & $65 \%$ & $40 \%$ \\
U 850 verano & $50 \%$ & $35 \%$ \\
V 200 U 850 verano & $65 \%$ & $35 \%$ \\
\hline
\end{tabular}

pendientes), el límite de aceptabilidad es de 15 aciertos (75\%) en midiles y $11(55 \%)$ en terciles.

Únicamente se encuentran porcentajes de acierto aceptables en primavera.

\subsubsection{Validación por Brier Score de modelos probabilísticos}

El criterio de aceptabilidad de esta medida establece como buenos valores a los BS menores a 0.500 para midiles y menores a 0.667 para terciles.

Los valores obtenidos se muestran en la Tabla 4.

En primavera, la combinación de predictores mejora los resultados de los modelos que predicen con un único predictor, siendo éste el único modelo bueno tanto en predicción de midiles como de terciles. Por otro lado, de acuerdo a esta validación, en verano V 200 es el mejor modelo.

\subsection{Caso particular: Área metropolitana de Montevideo}

Como caso particular, se adapta la metodología a la estación meteorológica de Carrasco, ubicada en el área metropolitana de Montevideo. A continuación se exponen los resultados obtenidos para verano ya que son mejores que para todo el sur del país. Por otro lado, en primavera la habilidad de los modelos es similar a la de los modelos del sur.

Los índices a utilizar como predictores son los mismos que en las secciones anteriores. Sin embargo, las re-

Tabla 4 - rier Score original. En negrita los valores buenos.

\begin{tabular}{lcc}
\hline Predictor & BS midiles & BS terciles \\
\hline U 200 primavera & 0.505 & 0.673 \\
V 200 primavera & $\mathbf{0 . 4 5 2}$ & 0.678 \\
V 850 primavera & 0.528 & 0.689 \\
U 200 V 850 primavera & $\mathbf{0 . 4 1 5}$ & $\mathbf{0 . 5 9 4}$ \\
U 200 verano & $\mathbf{0 . 4 7 4}$ & 0.830 \\
V 200 verano & $\mathbf{0 . 4 1 4}$ & 0.791 \\
U 850 verano & 0.559 & 0.811 \\
V 200 U 850 verano & $\mathbf{0 . 4 6 7}$ & 0.784 \\
\hline
\end{tabular}


gresiones se calculan en base a las observaciones de PP en la estación de Carrasco.

La Tabla 5 resume los resultados de las validaciones obtenidos en Carrasco, mostrando únicamente sus niveles de significancia.

En general, los resultados son mejores que los que se obtienen para todo el sur del país. Con respecto a la correlación índice-precipitación, los tres índices mantienen correlaciones de Pearson y Spearman estadisticamente significativas. En el caso de la correlación pronóstico-observación, tres de los modelos mantienen correlaciones significativas. También se destaca que si bien V 200 es el que ofrece los mejores resultados en esta validación, la combinación de $\mathrm{V}$ 200 y U 850 es aún mejor. En la validación por porcentaje de acierto, no se encuentra ningún modelo con buenos resultados tanto en midiles como terciles. Finalmente, los valores de BS en tres de los modelos son buenos. La combinación de los índices, además de mejorar los resultados de esta validación, disminuye significativamente el BS de midiles.

\section{Conclusiones}

El trabajo buscó desarrollar una metodología de pronóstico dinámico-estadístico para la precipitación estacional en el sur de Uruguay en primavera y verano que será la base para un pronóstico operativo. Para la parte dinámica se utilizó el modelo global ICTP-MCGA. Luego, se correlacionaron linealmente las salidas de vientos del modelo dinámico promediadas en diferentes alturas y regiones con la precipitación en el sur de Uruguay. Las medias espaciales de los vientos en las regiones que mantienen correlaciones estadísticamente significativas y con sentido físico con la precipitación en el sur de Uruguay son los índices que se utilizaron como predictores. La combinación de las salidas de ICTP-MCGA y los parámetros de regresión entre los índices y la precipitación en la zona de interés conforman los modelos de pronóstico. Estos modelos pronostican de manera determinística y probabilística.
El mejor índice predictor que se encontró es V 200. En particular, para verano en el sur de Uruguay, el modelo que predice con V 200 es el de mejor habilidad. Sin embargo, en primavera en el sur del país la combinación de los restantes predictores tiene mejores resultados, mientras que en verano en el área metropolitana de Montevideo, la combinación de V 200 y U 850 genera el mejor modelo. Considerando el mejor modelo para cada situación se encuentra que en verano las validaciones son buenas únicamente en correlación de Spearman de índice-PP y en BS midiles mientras que en primavera el pronóstico es bueno en las dos correlaciones índice-PP, en porcentaje de acierto de terciles y en BS midiles y terciles. Finalmente en verano en el área metropolitana de Montevideo el pronóstico es bueno en las dos correlaciones índice-PP, en porcentaje de acierto midiles y en BS midiles y terciles. En base a esto se concluye que el promedio de la zona sur es más pronosticable en primavera que en verano mientras que durante el verano es más pronosticable el área metropolitana de Montevideo que el promedio de la zona sur. Por otro lado, en primavera, se encontraron habilidades similares en los modelos para el área metropolitana de Montevideo y para todo el sur del país (no se muestra). El hecho de encontrar mejores pronósticos en el área metropolitana de Montevideo que en toda la zona sur en verano pero no en primavera es consistente con un análisis (que no se muestra), donde se destaca que la zona metropolitana de Montevideo es un máximo local de correlación con el Índice Niño 3 en verano pero un mínimo en primavera.

Finalmente, se concluye que, en general, V 200 es el mejor predictor. Como se mencionó anteriormente, la región en la que se promedia esta variable y su relación con la precipitación en Uruguay ha sido estudiada por varios autores (por ejemplo, Robertson y Mechoso, 2000, Cazes-Boezio, 2003). La región seleccionada para este índice caracteriza el anticiclón que domina la variabilidad de la circulación regional a escala interanual y que influencia las lluvias del sudeste de Sudamérica.

Tabla 5 - Resultados de las validaciones obtenidos en Carrasco. En negrita los valores buenos.

\begin{tabular}{|c|c|c|c|c|c|}
\hline Índice & & $\mathrm{U} 200$ & V 200 & $\mathrm{U} 850$ & V $200 \mathrm{U} 850$ \\
\hline \multirow[t]{2}{*}{ Significancia correlación índice-PP } & Pearson & $99 \%$ & $99 \%$ & $96 \%$ & No corresponde ${ }^{\#}$ \\
\hline & Spearman & $98 \%$ & $99 \%$ & $95 \%$ & No corresponde \\
\hline \multirow[t]{2}{*}{ Significancia correlación pronóstico-observación } & Pearson & $93 \%$ & $96 \%$ & NO & $97 \%$ \\
\hline & Spearman & $93 \%$ & $98 \%$ & NO & $98 \%$ \\
\hline \multirow[t]{2}{*}{ Porcentaje de acierto } & Midiles & $80 \%$ & $70 \%$ & $70 \%$ & $75 \%$ \\
\hline & Terciles & $40 \%$ & $60 \%$ & $40 \%$ & $35 \%$ \\
\hline \multirow[t]{2}{*}{ Brier Score } & Midiles & 0.393 & 0.402 & 0.400 & 0.285 \\
\hline & Terciles & 0.590 & 0.537 & 0.684 & 0.460 \\
\hline
\end{tabular}

\#Las correlaciones entre índice y precipitación para el caso de índices combinados no tienen sentido ya que estas correlaciones se calculan entre dos variables únicamente. 


\section{Referencias}

AMEZCUA, J.; KALNAY, E.; WILLIAMS, P.D. The effects of the raw filter on the climatology and forecast skill of the speedy model. Monthly Weather Review, v. 139, n. 2, p. 608-619, 2011.

BARREIRO, M. Influence of enso and the south Atlantic Ocean on climate predictability over southeastern south america. Climate Dynamics, v. 35, n. 7-8, p. 1493-1508, 2010.

BARREIRO, M.; TIPPMANN, A. Atlantic modulation of el niño influence on summertime rainfall over southeastern South America. Geophysical Research Letters, v. 35, n. 16, 2008.

CARVALHO, L.M.; JONES, C.; LIEBMANN, B. The south atlantic convergence zone: Intensity, form, persistence, and relationships with intraseasonal to interannual activity and extreme rainfall. Journal of Climate, v. 17, n. 1, p. 88-108, 2004.

CAZES-BOEZIO, G.; ROBERTSON, A.W.; MECHOSO, C.R. Seasonal dependence of enso teleconnections over South America and relationships with precipitation in Uruguay. Journal of Climate, v. 16, n. 8, p. 1159-1176, 2003.

CAZES-BOEZIO, G.; TALENTO, S.; PISCIOTTANO JALABERT, G.J. Seasonal probability forecasts of decemberjanuary-february precipitation in northern Uruguay and Rio Grande do Sul obtained with the coupled forecast system v2 of noaa and statistical downscaling. Revista Brasileira de Meteorologia, v. 27, n. 4, p. 377-387, 2012.

DEE, D.; UPPALA, S.; SIMMONS, A.; BERRISFORD, P.; POLI, P. et al. The era-interim reanalysis: Configuration and performance of the data assimilation system. Quarterly Journal of the Royal Meteorological Society, v. 137, n. 656, p. 553-597, 2011.

DIAZ, A.F.; STUDZINSKI, C.D.; MECHOSO, C.R. Relationships between precipitation anomalies in Uruguay and Southern Brazil and sea surface temperature in the Pacific and Atlantic Oceans. Journal of Climate, v. 11, n. 2, p. 251-271, 1998.

GRIMM, A.M.; BARROS, V.R.; DOYLE, M.E. Climate variability in southern South America associated with el niño and la niña events. Journal of Climate, v. 13, n. 1, p. 35-58, 2000.

HOLTON, J.R.; DMOWSKA, R. El niño, la niña, and the southern oscillation. Academic Press, 1989. 293p.

HOLTON, J.R.; HAKIM, G.J. An introduction to dynamic meteorology. Academic Press, 2012. 400p.

HOREL, J.D.; WALLACE, J.M. Planetary-scale atmospheric phenomena associated with the southern oscillation. Monthly Weather Review, v. 109, n. 4, p. 813-829, 1981.

KUCHARSKI, F.; MOLTENI, F.; BRACCO, A. Decadal interactions between the western tropical pacific and the north atlantic oscillation. Climate Dynamics, v. 26, n. 1, p. 79-91, 2006.

KUCHARSKI, F.; MOLTENI, F.; KING, M.P.; FARNETI, R.; KANG, I.S. et al. On the need of intermediate complexity general circulation models: a speedy example. Bulletin of the American Meteorological Society, v. 94, n. 1, p. 25-30, 2013.
LATIF, M.; ANDERSON, D.; BARNETT, T.; CANE, M.; KLEEMAN, R. et al. A review of the predictability and prediction of enso. Journal of Geophysical Research: Oceans, v. 103, n. C7, p. 14375-14393, 1998.

LORENZ, E.N. Deterministic nonperiodic flow. Journal of the Atmospheric Sciences, v. 20, n. 2, p. 130-141, 1963.

MASON, S.J. Guidance on verification of operational seasonal climate forecasts. World Meteorological Organization, Commission for Climatology XIV Technical Report, 2013.

CHAND, S.S.; WALSH, K.J. Modeling seasonal tropical cyclone activity in the Fiji region as a binary classification problem. Journal of Climate, v. 25, n. 14, p. 5057-5071, 2012.

MOLTENI, F. Atmospheric simulations using a gem with simplified physical parametrizations. i: Model climatology and variability in multidecadal experiments. Climate Dynamics, v. 20, n. 2, p. 175-191, 2003.

PICASSO, V.; CRUZ, G.; ASTIGARRAGA, L.; TERRA, R. Cambio y variabilidad climática. Espacio Interdisciplinario, Universidad de la República, 2012. 122p.

PISCIOTTANO, G.; DÍAZ, A.; CAZESS, G.; MECHOSO, C.R. El niño-southern oscillation impact on rainfall in Uruguay. Journal of Climate, v. 7, n. 8, p. 1286-1302, 1994.

ROBERTSON, A.W.; MECHOSO, C.R. Interannual and interdecadal variability of the south atlantic convergence zone. Monthly Weather Review, v. 128, n. 8, p. 2947-2957, 2000.

ROPELEWSKI, C.F.; HALPERT, M.S. Global and regional scale precipitation patterns associated with the el niño/southern oscillation. Monthly Weather Review, v. 115, n. 8, p. 1606-1626, 1987.

SALIO, P.; NICOLINI, M.; ZIPSER, E.J. Mesoscale convective systems over southeastern South America and their relationship with the south american low-level jet. Monthly Weather Review, v. 135, n. 4, p. 1290- 1309, 2007.

SAHA, S.; MOORTHI, S.; WU, X.; WANG, J.; NADIGA, S. et al. The ncep climate forecast system version 2. Journal of Climate, v. 27, n. 6, p. 2185-2208, 2014.

STEIN, M.L. Interpolation of spatial data: some theory for kriging. Springer Science \& Business Media, 2012. 2493p.

TROCCOLI, A. Seasonal climate forecasting. Meteorological Applications, v. 17, n. 3, p. 251-268, 2010.

UPPALA, S.; DEE, D.; KOBAYASHI, S.; BERRISFORD, P.; SIMMONS, A. Towards a climate data assimilation system: Status update of era-interim. ECMWF Newsletter, 115, 12-18, 2008.

WANG, B. Interdecadal changes in el niño onset in the last four decades. Journal of Climate, v. 8, n. 2, p. 267-285, 1995.

WILKS, D.S. Statistical methods in the atmospheric sciences. Academic press. Amsterdam., 2011. v. 100.

ZAMBONI, L.; MECHOSO, C.R.; KUCHARSKI, F. Relationships between upper-level circulation over South America and rainfall over southeastern South America: A physical base for seasonal predictions. Journal of Climate, v. 23, n. 12 , p. $3300-3315,2010$.

This is an Open Access article distributed under the terms of the Creative Commons Attribution Non-Commercial License which permits unrestricted non-commercial use, distribution, and reproduction in any medium provided the original work is properly cited. 